\section{The role of protein arginine methylation in the formation of silent chromatin}

\author{
Michael C. Yu, ${ }^{1,3}$ Dudley W. Lamming, ${ }^{2}$ \\ Julian A. Eskin, ${ }^{1}$ David A. Sinclair, ${ }^{2,5}$ \\ and Pamela A. Silver ${ }^{1,4}$ \\ ${ }^{1}$ Department of Systems Biology, Harvard Medical School, \\ Boston, Massachusetts 02115, USA; ${ }^{2}$ Department \\ of Pathology, Harvard Medical School, \\ Boston, Massachusetts 02115, USA
}

Establishment and maintenance of silent chromatin in the Saccharomyces cerevisiae involves a step-wise assembly of the SIR complex. Here we demonstrate a role for the protein arginine methyltransferase Hmt1 in this process. In the absence of catalytically active Hmt1, yeast cells display increased transcription from silent chromatin regions and increased mitotic recombination within tandem repeats of rDNA. At the molecular level, loss of Hmt1's catalytic activity results in decreased Sir2 and dimethylated Arg-3 histone $\mathrm{H} 4$ occupancy across silent chromatin regions. These data suggest a model whereby protein arginine methylation affects the establishment and maintenance of silent chromatin.

Received April 11, 2006; revised version accepted October 23, 2006.

In a eukaryotic cell, different chromosomal regions display varying degrees of transcriptional competency. This is the result of selective transcriptional repression or silencing that renders specific chromatin domains inaccessible to the transcriptional machinery. Analogous to metazoan heterochromatin, there are three chromosomal regions in the yeast Saccharomyces cerevisiae that are epigenetically transcriptionally silenced (Rusche et al. 2003); the telomeres, the silent mating loci (HMR and $H M L)$, and the rDNA loci. Within these regions are cisacting elements known as silencers and telomeric repeats that serve as nucleation points to recruit transacting proteins responsible for the establishment and maintenance of silent chromatin.

One of the most prominent trans-acting proteins involved in the establishment and maintenance of silencing at these loci is Silent Information Regulator-2 (Sir2), an $\mathrm{NAD}^{+}$-dependent histone deacetylase (Blander and Guarente 2004). Sir2 plays a crucial role in the formation of silent chromatin. In addition, Sir2 is involved in the maintenance of genome stability via the repair of doublestranded DNA breaks by nonhomologous recombina-

[Keywords: Hmt1; Sir2; arginine methylation; silent chromatin] ${ }^{3}$ Present address: Department of Biological Sciences, 109 Cooke Hall, State University of New York at Buffalo, Buffalo, NY 14260, USA. Corresponding authors.

${ }^{4}$ E-MAIL pamela_silver@hms.harvard.edu; FAX (617) 432-6405.

${ }^{5}$ E-MAIL david_sinclair@hms.harvard.edu; FAX (617) 432-3932.

Article is online at http://www.genesdev.org/cgi/doi/10.1101/gad.1495206. tion. For example, Sir2 is recruited to sites of DNA strand breaks (Martin et al. 1999; Mills et al. 1999) and Sir2-dependent localized histone acetylation triggers the homologous recombination pathway of double-strand DNA repair (Tamburini and Tyler 2005).

Sir2 forms complexes with different proteins to promote silencing. For example, the Sir $1 / 2 / 3 / 4$ complex functions to silence transcription at the mating-type loci, whereas a Sir2/3/4 complex mediates silencing at the telomeres (Kaeberlein et al. 1999). At the rDNA, Sir2 associates with Cdc14 and Net1 to form the RENT (regulator of the nucleolar silencing and telophase exit) complex (Ghidelli et al. 2001). These complexes are thought to promote the formation of silent chromatin through stepwise propagation along the DNA (Hoppe et al. 2002).

Arginine methylation is a post-translational modification commonly found in nucleic acid-binding proteins (Bedford and Richard 2005). The enzyme that catalyzes this modification belongs to a growing enzyme family called protein arginine methyltransferases or PRMTs. Arginine residues may be either monomethylated or dimethylated by PRMTs. The type I enzymes catalyze the formation of asymmetric dimethylarginines, whereas type II enzymes facilitate the formation of symmetric dimethylarginines (McBride and Silver 2001; Bedford and Richard 2005). Once thought to be a static modification, the recent discovery of PAD4, an enzyme that converts monomethylated arginine into citrulline (Wang et al. 2004), demonstrates that arginine methylation can, in fact, be dynamic.

Arginine methylation regulates its substrate's function by modulating its protein-protein interaction. For example, loss of arginine methylation on the STAT1 protein inhibits its association with its inhibitor PIAS, thereby causing a decreased interferon response (Mowen et al. 2001). The methylation status of the Src kinase substrate Sam68 controls its ability to interact with SH3-containing proteins, and thereby affects its function (Bedford et al. 2000). Additionally, arginine methylation of $\mathrm{Spt} 5$ regulates its interaction with RNA polymerase II (Pol II) and this interaction is crucial for proper transcription (Kwak et al. 2003).

Hmt1 (also termed Rmt1) has been identified as the major type I arginine methyltransferase in yeast, and is the functional homolog of mammalian PRMT1 (Gary et al. 1996; Henry and Silver 1996). One of the earliest functions identified for Hmtl was its ability to modulate nucleo-cytoplasmic shuttling for a number of RNAbinding proteins (RBPs) involved in the biogenesis of messenger ribonucleoparticles (mRNPs) (Shen et al. 1998). Hmt1 also influences mRNP dynamics by modulating protein-protein interactions and recruitment of mRNA processing and export factors (Yu et al. 2004). Additionally, Hmt1 is cotranscriptionally recruited to genes (Yu et al. 2004), raising the possibility that Hmt1 functions as a regulator of gene expression.

In this study, we demonstrate that protein arginine methylation plays a novel role in the preservation and establishment of silent chromatin domains. In the absence of Hmtl activity, we observe an increase in the rate of mitotic recombination within the rDNA array, and overexpression of $\mathrm{Hmt} 1$ decreases the rate of mitotic recombination. Furthermore, we have used chromatin 
immunoprecipitation (ChIP) to illustrate the importance of Hmt1's activity for the targeted recruitment of Sir2 to silent chromatin domains. Finally, we show a localized decrease in the silent chromatin occupancy for dimethylated Arg-3 histone H4, an in vitro substrate of Hmt1. Together, these results demonstrate a novel function for arginine methylation in the maintenance of genome stability and the establishment of silent chromatin.

\section{Results and Discussion}

Transcriptional profiling of both the null and the catalytic mutants of $\mathrm{Hmtl}$ indicated no major changes in overall gene expression (Yu et al. 2004). Nevertheless, a small number of transcripts were more abundant in the loss-of-function Hmt1 mutants than in wild type. Subsequently, we determined that these transcripts correspond to regions proximal to or within regions of epigenetically maintained silent chromatin (Table 1). This observation suggests that $\mathrm{Hmt} 1$ may play a role in regulating the establishment and maintenance of silent chromatin.

To test this hypothesis, we used a silencing assay to measure silencing in hmt1s mutants. The ADE2-based phenotypic assay (Anderson et al. 2002), in which ADE2 is inserted at telomeric regions, indicated a loss of silencing at the telomeric regions in the $h m t 1 \Delta$ mutants (Fig. 1A). This result is consistent with the microarray data, in which we detected an increased abundance of transcripts encoded by genes located near the telomeric regions in $h m t 1 \Delta$ mutants.

Hmtl is also required for the maintenance of genome stability. Transcriptional silencing is normally observed in heterochromatin, and heterochromatin has been shown to regulate cellular recombination events (Lesage and Todeschini 2005). Therefore, we measured homologous recombination rate between tandem repeats within the rDNA array in cells bearing hmt1 mutations to determine whether there was an overall change in genomic stability. This assay involved measuring the rate of mitotic intrachromosomal recombination within the rDNA repeat array by monitoring the loss of the inserted $A D E 2$ marker during the first cell division after plating (see Materials and Methods). The frequency of rDNA recombination increased more than twofold versus wild type in both hmt1s (Fig. 1B) and a previously generated Hmt1 catalytic (G68R) mutant (Fig. 1C; McBride et al. 2000). We next sought to determine if the rate of rDNA mitotic recombination could be lowered by overexpressing Hmt1. Using yeast cells that overexpressed Hmt1 under the constitutive alcohol dehydrogenase ADH1 promoter, we observed that Hmtl overexpression de-

Table 1. Identity of abundant transcripts that are initiated from within or next to silent chromatin domains in Hmt1 catalytic mutants when compared with wild-type cells

Telomere-proximal regions

YHL047C, YCR102C, YAR064W, YAR071W

Silent mating loci

YCL066W, YCL065W, YCL067C, YCR096C

rDNA regions

YLR158C, YLR160C, YLR157C, YLR155C, YLR159W
A.

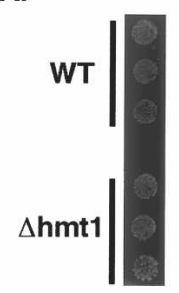

B.

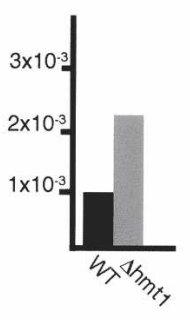

D.

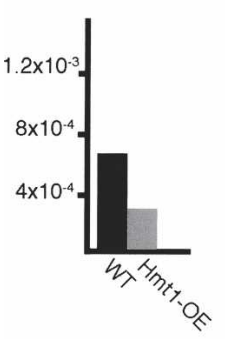

E.

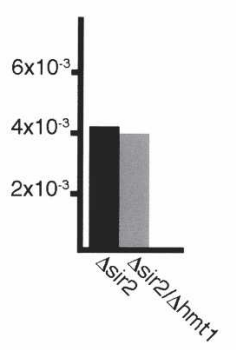

C.

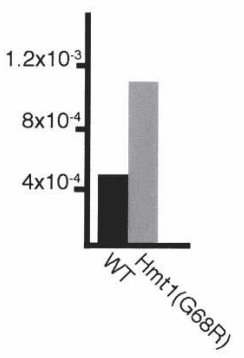

F.

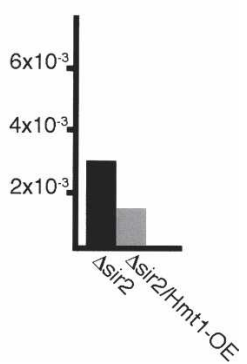

Figure 1. Protein arginine methylation affects genome stability. The mitotic rDNA recombination rate was measured to assess the degree of genomic instability in Hmt1 mutants. The frequency of the loss of an $A D E 2$ marker was calculated from at least three clones totaling $>40,000$ colonies. The fold of change as well as the standard error (SE) were calculated in each experiment. $(A)$ Silencing assay showed increased loss of telomeric silencing in $h m t 1 \Delta$ mutants. Mitotic recombination assay showed wild-type versus $h m t 1 \Delta$ mutants (2.1-fold increase with SE of 0.1) (B), wild-type versus Hmt1 catalytic mutants (G68R) (2.4-fold increase with SE of 0.4$)(C)$, wildtype versus Hmtl-overexpressing mutants (2.2-fold decrease with $\mathrm{SE}$ of 0.3$)(D)$, sir2 $\Delta$ mutant versus sir2 $\Delta / h m t 1 \Delta$ (onefold with SE of $0.2)(E)$, and sir2s versus sir2s with Hmt1-overexpressing background (1.83-fold decrease with SE of 0.1$)(F)$.

creased the frequency of rDNA mitotic recombination by greater than twofold (Fig. 1D). In sum, transcriptional profiling, silencing assays, and an rDNA mitotic recombination assay all point to a role for Hmtl in the maintenance of genome stability.

Since Sir2 has a well-known role in the establishment of silent chromatin formation in S. cerevisiae, we tested the role of Hmt1 in Sir2-mediated pathways by measuring the rDNA mitotic recombination rate in various combinations of sir $2 \Delta$ and $h m t 1 \Delta$ mutant backgrounds. We observed no significant change in the rate of rDNA mitotic recombination between a sir2 $\Delta$ and a dual sir2 $\Delta /$ $h m t 1 \Delta$ strain (Fig. 1E). In contrast, a Sir2-overexpressing/ $h m t 1 \Delta$ strain exhibited a twofold decrease in mitotic recombination rate when compared with a Sir2-overexpression/HMT1 strain (data not shown). This observation suggests that $\mathrm{Hmtl}^{\prime}$ s role in regulating rDNA recombination is dependent upon Sir2.

We next wanted to determine whether overexpressing Hmt1 in the absence of Sir2 had any affect on the rate of mitotic recombination. Interestingly, we observed a greater than twofold decrease in the rDNA mitotic recombination rate when we compared a sir $2 \Delta / \mathrm{Hmt} 1$ overexpressing strain to a sir2 $\Delta$ only strain (Fig. 1 F). This result indicates that overexpression of Hmtl can suppress the rate of rDNA mitotic recombination in the absence of Sir2. These data point to the existence of an 
alternative, Sir2-independent pathway in genome preservation that is affected by Hmt1's function.

Given that protein arginine methylation often affects protein-protein interactions (Bedford and Richard 2005), we used ChIP to determine whether the recruitment of Sir2 to silent chromatin regions was affected in various Hmt1 mutants. We designed primers against telomeric regions known to contain Sir2 (Fig. 2A; Hoppe et al. 2002). At telomeres, Sir2 occupancy in a strain expressing a catalytic mutant of Hmt1 (G68R) was found to be less than in the wild-type cells (Fig. 2A, cf. dark-gray and black bars). The most pronounced defect was observed at the region closest to the end of telomere (Fig. 2A, primer set A), followed by a gradual, smaller change distal to the end of telomere (Fig. 2A). Interestingly, Sir2 occupancy is higher in the Hmt1-overexpressing mutants than in wild type (Fig. 2A, light-gray bars), indicating that overexpression of Hmt1 promotes the recruitment of Sir2 to the telomeric region.

A.
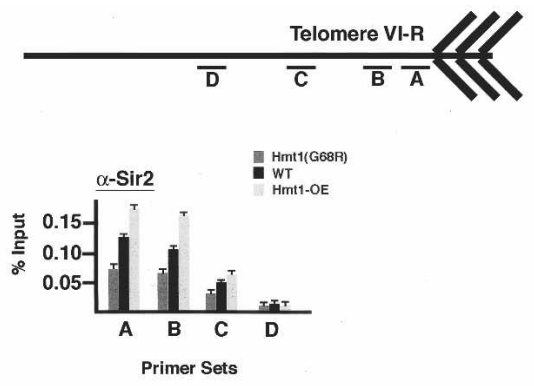

B.
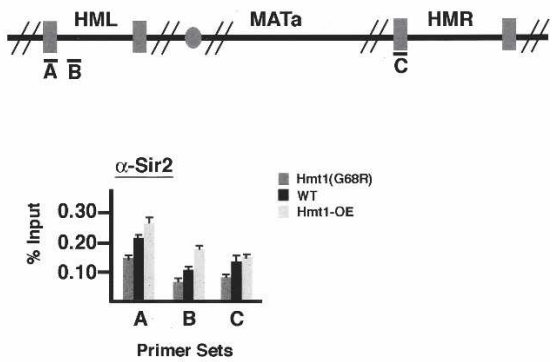

c.
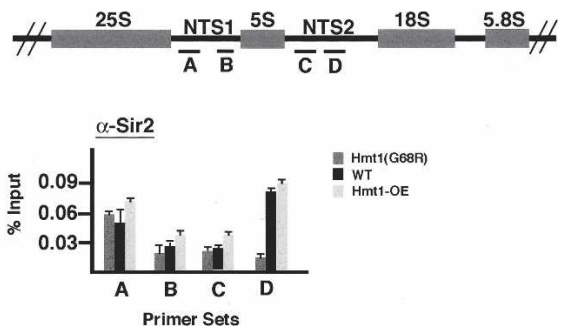

Figure 2. Sir2 occupancy across silent chromatin regions. ChIP was carried out in wild-type, Hmt1 catalytic mutant (G68R), and Hmt1overexpressing mutant strains using anti-Sir2 antibody. Schematic representations of the silent chromatin regions interrogated in this study are shown with indication of primer sets used in quantitative PCR experiments. $(A)$ Telomere VI-R region. (B) Silent mating loci. $(C)$ rDNA locus. The results from quantitative PCR on immunoprecipitated DNA from all three strains are shown in bar graphs. The color of the bar graphs indicates the strain used. (Black) Wild-type; (dark gray) Hmt1(G68R); (light gray) Hmtl-overexpressing mutants. The letter below each graph corresponds to the primer set used for amplification according to the respective schematic diagram.
We extended our analysis by comparing the levels of Sir2 occupancy across the silent mating loci in Hmt1 mutants versus wild-type cells (Fig. 2B; Hoppe et al. 2002). Similar to the trend observed in the telomeric regions, the Hmt1 catalytic mutants exhibited an approximately twofold decrease in Sir2 recruitment to silent mating loci compared with the wild type (Fig. 2B, cf. dark-gray and black bars). Similar to what was observed at the telomeres, Sir2 recruitment was slightly increased in the Hmt1-overexpressing mutants than in the wildtype cells (Fig. 2B, cf. light-gray and black bars).

Since the transcriptional silencing of rDNA repeats requires Sir2 (Hoppe et al. 2002), we also examined the effect of Hmt1 on Sir2 occupancy across nontranscribed spacer regions of the ribosomal DNA locus, NTS-1 and NTS-2 (Fig. 2C). Sir2 occupancy was unchanged in the catalytic or the Hmtl-overexpressing mutants when compared with that of the wild type in all regions except the $3^{\prime}$ end of the NTS-2 (Fig. 2C). In the Hmtl catalytic mutants, Sir2 occupancy at the 3' end of NTS-2 region was approximately threefold less than in wild type (Fig. $2 \mathrm{C}$, primer set $\mathrm{D}$, cf. dark-gray and black bars). In the Hmt1-overexpressing mutants, Sir2 occupancy remained at about the same level as that in wild-type cells (Fig. 2C, primer set D, cf. light-gray and black bars).

Upon binding to DNA, Sir2 deacetylates histones in vivo (Landry et al. 2000). Thus, we measured the effect of inactivating or overexpressing $\mathrm{Hmt} 1$ on the acetylation status of histone tails across the silent chromatin regions. Given that Sir2 recruitment was compromised in the Hmt1 catalytic mutants, we wanted to determine if this effect translates into changes in the acetylation status of histone tails across the silent chromatin regions. To this end, we used antibodies that recognized the acetylated tail of histone $\mathrm{H} 4$ to perform ChIP across telomeric regions and silent mating loci. At the telomeres, the levels of acetylated histone $\mathrm{H} 4$ increased in the catalytic mutant, with the highest levels of acetylated histone $\mathrm{H} 4$ observed in regions most distal to the telomere ends (Fig. 3A, telomere VI-R panel). In contrast, the overexpression of Hmt1 had little effect on $\mathrm{H} 4$ acetylation at telomeres (Fig. 3A). At the silent mating HML loci, acetylated histone $\mathrm{H} 4$ was higher in the catalytic mutant than in wild type, and lower still in cells overexpressing Hmt1 (Fig. 3B, mating loci panel). Overall, the levels of acetylated histone $\mathrm{H} 4$ occupancy in the Hmtl catalytic mutant and in cells overexpressing Hmtl reflect the changes observed with the Sir2 occupancy in these same mutants (cf. Figs. 2 and 3). Since the status of histone acetylation corresponds to transcriptional competency, the observed increase in acetylated histone $\mathrm{H} 4$ occupancy in the Hmtl catalytic mutants supported the observed increase in transcriptional activities across these regions in these mutants.

Since histone modification has been shown to be important for its subsequent interactions with the transcriptional machinery (Morillon et al. 2003) and Hmt1 can methylate Arg-3 on histone $\mathrm{H} 4$ in vitro (Lacoste et al. 2002), we examined the status of dimethylated Arg-3 $\mathrm{H} 4$ occupancy at the silent chromatin region using antidimethyl-histone H4 antibody (Strahl et al. 2001). The trend of dimethyl-Arg-3H4 occupancy at the telomeres was similar to that of Sir2: Dimethyl-Arg-3H4 decreased approximately twofold in the catalytic mutants when compared with wild type (Fig. 4A, cf. dark-gray and black bars). The extent of this change decreased with distance 
Yu et al.

A.

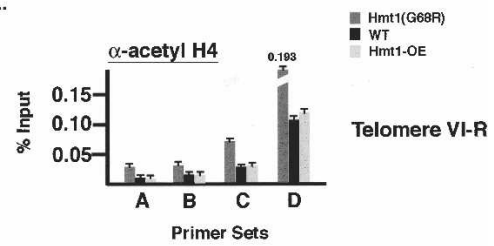

B.

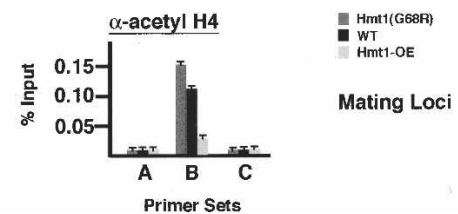

Figure 3. Acetylated histone H4 occupancy across silent chromatin regions. ChIP was carried out from wild-type, Hmtl catalytic mutant (G68R), and Hmt1-overexpressing mutant strains using anti-acetylated histone $\mathrm{H} 4$ antibody. Quantitative PCR was performed using primer sets identical to those used in Figure 2, and results from the three strains are shown in the bar graphs. The letter below each graph corresponds to the amplified sequence according to the respective schematic diagram.

from the end of the telomere (Fig. 4A). Overexpression of $\mathrm{Hmt1}$ significantly enhanced dimethyl-Arg-3H4 occupancy when compared with wild type (Fig. 4A, cf. lightgray and black bars), suggesting that overexpression of $\mathrm{Hmt1}$ promotes arginine methylation of histone $\mathrm{H} 4$ at telomeric regions. A similar trend was observed at the silent mating loci (Fig. 4B), in which dimethyl-Arg-3H4 occupancy was decreased in the Hmtl catalytic mutant and paralleled the occupancy of Sir2.

In contrast to the mating-type loci and telomeres, dimethyl-Arg-3H4 occupancy across nontranscribed spacer regions of the ribosomal DNA locus exhibited a different profile than Sir2 binding. The downstream portion of the NTS-1 region is the only region where dimethyl-Arg-3H4 occupancy is lower than in wild type (Fig. 4C, primer set B). Interestingly, dimethyl-Arg-3H4 occupancy was lower than wild type in both the catalytic and the overexpressing mutant at both regions of the NTS-2 (Fig. 4C, primer sets C and D). In sum, the occupancy of dimethyl-Arg-3H4 is compromised at regions of silent chromatin, with the most pronounced defect at the telomeres and silent mating loci.

How might protein arginine methylation affect rDNA mitotic recombination? Since Sir2 localizes to the vicinity of the rDNA ARS, decreased Sir2 recruitment to the ARS-containing NTS-2 region might affect the higherorder chromatin structure of rDNA given the known histone deacetylase activity of Sir2. One possible scenario is that the absence of Sir2-mediated deacetylation results in the unfolding of rDNA chromatin, making it more accessible to recombination events. Evidence for this hypothesis comes from the demonstration that rDNA chromatin structure is altered in a Sir2-null strain (Fritze et al. 1997).

The connection between arginine methylation and genome maintenance has been recently examined in mammalian cells. It has been shown that arginine methylation regulates the molecular machinery involved in the DNA repair process. For example, PRMT1 methylates MRE11, a component of the MRE11/RAD50/NBS1
(MRN) complex that is known to be involved in the DNA damage response (Boisvert et al. 2005). Furthermore, cells lacking methylated MRE11 exhibit intra-Sphase DNA damage checkpoint defects (Boisvert et al. 2005).

Taken together, our data support a model in which arginine methylation adversely affects the genome stability via compromising the recruitment of Sir2 to the silent chromatin regions (Fig. 4D). In the absence of arginine methylation, Sir2 recruitment is decreased across all silent chromatin regions. This may be explained in part by the loss of arginine methylation at position 3 of histone $\mathrm{H} 4$, since this modification stimulates further histone modifications (Huang et al. 2005), which are important for specific interactions between histones and components of transcriptional machinery (Mellor 2006). For example, the acetylation of the $\mathrm{N}$ terminus of histone $\mathrm{H} 4$ regulates its interaction with Sir3 (Carmen et al. 2002). In addition, our data showing Hmtl's effect on specific, localized histone $\mathrm{H} 4$ supports a previous observation in which the loss of Hmt1 did not significantly alter the bulk signal of Arg3 modification of H4 in vivo, despite the fact it was a clear substrate in vitro (Lacoste et al. 2002). Nevertheless, Arg3 H4 has been previously

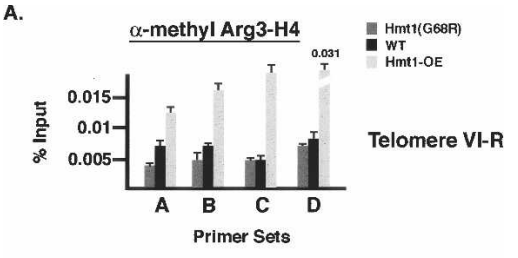

B.

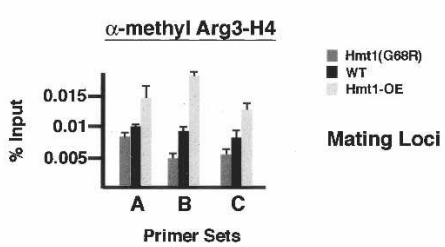

c.

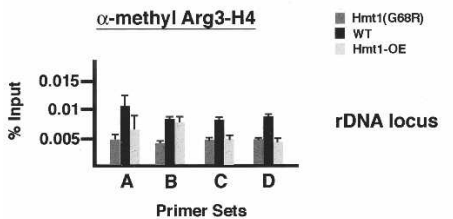

D

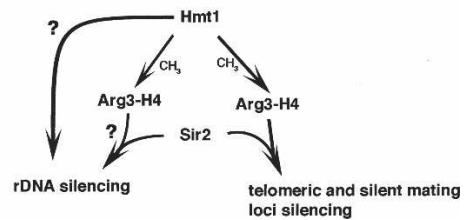

Figure 4. Dimethylated Arg-3 histone H4 occupancy across silent chromatin regions. ChIP was carried out from wild-type, Hmtl catalytic mutant (G68R), and Hmt1-overexpressing mutant strains using anti-dimethyl Arg-3 histone H4 antibody. Quantitative PCR was carried out using primer sets identical to those used in Figure 2, and results from the three strains are shown in the bar graphs. The letter below each graph corresponds to the amplified sequence according to the schematic representation diagram in telomeres $(A)$, silent mating loci $(B)$, and rDNA $(C) .(D)$ The proposed model for a role of protein methylation in genome maintenance. 
reported to be associated with transcriptional activation as opposed to transcriptional silencing (Huang et al. 2005). One plausible explanation is that the specific chromosomal context at which methylation of Arg3 H4 occurs influences the recruitment of activation or repression complex.

It is less clear as to how dimethylated Arg3H4 negatively affects Sir2 recruitment at the rDNA repeats, in particular within the NTS-2 region. Interestingly, cells overexpressing Hmtl display a lowered rate of mitotic rDNA recombination in the absence of Sir2. This indicates that Hmt1 may play a role in a different pathway that also promotes genomic stability. One possibility is that Hmt1 modulates the expression of stress-response genes since the genome-wide localization of Hmt1 revealed many stress-response genes bound by $\mathrm{Hmtl}$ (Yu et al. 2004). For example, overexpressing Hmt1 may somehow promote a DNA-damage response and thereby result in the decreased rate of recombination in the absence of Sir2. Alternatively, it is possible that loss of methylation actually promotes the recruitment of Sir2 homologues such as Hst1 and Hst2, which have recently been shown to be capable of suppressing rDNA recombination in the absence of Sir2 (Lamming et al. 2005).

\section{Materials and methods}

Yeast strains used in this study

All yeast strains used in this study are listed as follows: W303AR5 (MATa, ade2-1, leu2-3, can1-100, trp1-1, ura3-52, his3-11,15, RDN1::ADE2, RAD5), PSY 3424 (MATa, ade2-1, leu2-3, can1-100, trp11, ura3-52, his3-11,15, RDN1::ADE2, hmt1::KANMX6, RAD51, PSY3425 (MATa, ade2-1, leu2-3, can1-100, trp1-1, ura3-52, his3-11,15, RDN1::ADE2, hmt1::KANMX6, hmt1-G68R::LEU2, RAD5), PSY3426 (MATa, ade2-1, leu2-3, can1-100, trp1-1, ura3-52, his3-11,1,5, RDN1::ADE2, LEU2/HMT1, RAD5), YDS878 (MATa, ade2-1, leu2-3, can1-100, trp1-1, ura3-52, his3-11,15, RDN1::ADE2, sir2::TRP1, RAD51, PSY3427 (MATa, ade2-1, leu2-3, can1-100, trp1-1, ura3-52, his3-11,15, RDN1::ADE2, sir2::TRP1, Hmt1::KANMX6, RAD5), PSY3428 (MATa, ade2-1, leu2-3, can1-100, trp1-1, ura3-52, his3-11,15, RDN1::ADE2,

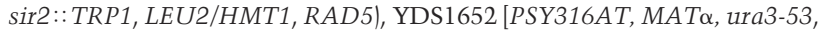
leu2-3, 112, ade2-101, can1-100, ADE2-TEL V-R, RDN1(18S)::URA3], and PSY3434 [MAT $\alpha$, ura3-53, 1eu2-3, 112, ade2-101, can1-100, ADE2TEL V-R, RDN1(18S)::URA3, hmt1::KANMX6]. Cells were grown at $30^{\circ} \mathrm{C}$ on YPD medium ( $1 \%$ yeast extract, $2 \%$ bactopeptone, $2 \%$ glucose, $\mathrm{w} / \mathrm{v}$ ) unless otherwise stated.

Silencing and mitotic rDNA recombination assay

The silencing assays were carried out as previously described (Bitterman et al. 2002). The extent of silencing at the telomeric region was determined using $A D E 2$-based telomeric silencing assay as described previously (Anderson et al. 2003). Mitotic rDNA recombination rates were determined as described previously (Christman et al. 1988). The frequency of loss of an ADE2 marker integrated into the rDNA array was used to measure recombination. Strains were grown to mid-log phase in liquid YPD medium, diluted, and plated onto solid YPD. Colonies were grown for $48 \mathrm{~h}$ at $30^{\circ} \mathrm{C}$ and then transferred to $4^{\circ} \mathrm{C}$ for $3 \mathrm{~d}$ prior to analysis. Half-sectored red and white colonies indicated a loss of ADE2 marker during the first cell division after plating. The rate of recombination was determined by counting the number of first cell division after plating and was calculated by dividing the total number of half-sectored colonies by the total number of colonies. At least 40,000 total colonies performed in triplicate were counted in each experiment, using Bio-Rad Quantity One software.

ChIP

ChIPs in biological duplicates were performed as previously described (Lei and Silver 2002). For immunoprecipitations, polyclonal $\alpha$-Sir2 (kind gift from D. Moazed, Harvard Medical School, Boston, MA) antibody was precoupled to protein A-sepharose beads for each immunoprecipitation, followed by extensive washing. In the case of acetylated histone $\mathrm{H} 4$ or dimethyl-Arg3 histone H4, polyclonal $\alpha$-acetyl H4 (catalog \#06-598; Upstate Biotechnology) antibody or polyclonal $\alpha$-dimethyl Arg-3 H4 (catalog \#07-213; Upstate Biotechnology) antibody was precoupled to protein Asepharose beads.

\section{Acknowledgments}

We thank G. Adelmant, C. Brown, D. Drubin, J. Hurt, S. Komili, A. McBride, and M. Moore for critical reading of the manuscript; D. Moazed for the anti-Sir2 antibody; and members of the Sinclair and Silver laboratory for helpful discussions. M.C.Y. was supported by an NIH postdoctoral fellowship. D.L. was supported by a National Eye Institute training grant. This work was supported by grants from the NIH to D.A.S. and P.A.S.

\section{References}

Anderson, R.M., Bitterman, K.J., Wood, J.G., Medvedik, O., Cohen, H., Lin, S.S., Manchester, J.K., Gordon, J.I., and Sinclair, D.A. 2002. Manipulation of a nuclear $\mathrm{NAD}^{+}$salvage pathway delays aging without altering steady-state NAD ${ }^{+}$levels. J. Biol. Chem. 277: 18881-18890.

Anderson, R.M., Bitterman, K.J., Wood, J.G., Medvedik, O., and Sinclair, D.A. 2003. Nicotinamide and PNC1 govern lifespan extension by calorie restriction in Saccharomyces cerevisiae. Nature 423: 181185.

Bedford, M.T. and Richard, S. 2005. Arginine methylation an emerging regulator of protein function. Mol. Cell 18: 263-272.

Bedford, M.T., Frankel, A., Yaffe, M.B., Clarke, S., Leder, P., and Richard, S. 2000. Arginine methylation inhibits the binding of proline-rich ligands to Src homology 3, but not WW, domains. J. Biol. Chem. 275: 16030-16036.

Bitterman, K.J., Anderson, R.M., Cohen, H.Y., Latorre-Esteves, M., and Sinclair, D.A. 2002. Inhibition of silencing and accelerated aging by nicotinamide, a putative negative regulator of yeast sir2 and human SIRT1. J. Biol. Chem. 277: 45099-45107.

Blander, G. and Guarente, L. 2004. The Sir2 family of protein deacetylases. Annu. Rev. Biochem. 73: 417-435.

Boisvert, F.M., Dery, U., Masson, J.Y., and Richard, S. 2005. Arginine methylation of MRE11 by PRMT1 is required for DNA damage checkpoint control. Genes \& Dev. 19: 671-676.

Carmen, A.A., Milne, L., and Grunstein, M. 2002. Acetylation of the yeast histone $\mathrm{H} 4 \mathrm{~N}$ terminus regulates its binding to heterochromatin protein SIR3. J. Biol. Chem. 277: 4778-4781.

Christman, M.F., Dietrich, F.S., and Fink, G.R. 1988. Mitotic recombination in the rDNA of $S$. cerevisiae is suppressed by the combined action of DNA topoisomerases I and II. Cell 55: 413-425.

Fritze, C.E., Verschueren, K., Strich, R., and Easton Esposito, R. 1997. Direct evidence for SIR2 modulation of chromatin structure in yeast rDNA. ЕMBO J. 16: 6495-6509.

Gary, J.D., Lin, W.J., Yang, M.C., Herschman, H.R., and Clarke, S. 1996. The predominant protein-arginine methyltransferase from Saccharomyces cerevisiae. J. Biol. Chem. 271: 12585-12594.

Ghidelli, S., Donze, D., Dhillon, N., and Kamakaka, R.T. 2001. Sir2p exists in two nucleosome-binding complexes with distinct deacetylase activities. EMBO J. 20: 4522-4535.

Henry, M.F. and Silver, P.A. 1996. A novel methyltransferase (Hmtlp) modifies poly(A) ${ }^{+}-$RNA-binding proteins. Mol. Cell. Biol. 16: $3668-$ 3678.

Hoppe, G.J., Tanny, J.C., Rudner, A.D., Gerber, S.A., Danaie, S., Gygi, S.P., and Moazed, D. 2002. Steps in assembly of silent chromatin in yeast: Sir3-independent binding of a Sir2/Sir4 complex to silencers and role for Sir2-dependent deacetylation. Mol. Cell. Biol. 22: $4167-$ 4180.

Huang, S., Litt, M., and Felsenfeld, G. 2005. Methylation of histone H4 by arginine methyltransferase PRMT1 is essential in vivo for many subsequent histone modifications. Genes \& Dev. 19: 1885-1893.

Kaeberlein, M., McVey, M., and Guarente, L. 1999. The SIR2/3/4 complex and SIR2 alone promote longevity in Saccharomyces cerevisiae by two different mechanisms. Genes \& Dev. 13: 2570-2580.

Kwak, Y.T., Guo, J., Prajapati, S., Park, K.J., Surabhi, R.M., Miller, B., Gehrig, P., and Gaynor, R.B. 2003. Methylation of SPT5 regulates its 
Yu et al.

interaction with RNA polymerase II and transcriptional elongation properties. Mol. Cell 11: 1055-1066.

Lacoste, N., Utley, R.T., Hunter, J.M., Poirier, G.G., and Cote, J. 2002. Disruptor of telomeric silencing- 1 is a chromatin-specific histone H3 methyltransferase. J. Biol. Chem. 277: 30421-30424.

Lamming, D.W., Latorre-Esteves, M., Medvedik, O., Wong, S.N., Tsang, F.A., Wang, C., Lin, S.J., and Sinclair, D.A. 2005. HST2 mediates SIR2-independent life-span extension by calorie restriction. Science 309: 1861-1864.

Landry, J., Sutton, A., Tafrov, S.T., Heller, R.C., Stebbins, J., Pillus, L., and Sternglanz, R. 2000. The silencing protein SIR2 and its homologs are NAD-dependent protein deacetylases. Proc. Natl. Acad. Sci. 97: 5807-5811.

Lei, E.P. and Silver, P.A. 2002. Intron status and $3{ }^{\prime}$-end formation control cotranscriptional export of mRNA. Genes \& Dev. 16: 2761-2766.

Lesage, P. and Todeschini, A.L. 2005. Happy together: The life and times of Ty retrotransposons and their hosts. Cytogenet. Genome Res. 110: 70-90.

Martin, S.G., Laroche, T., Suka, N., Grunstein, M., and Gasser, S.M. 1999. Relocalization of telomeric Ku and SIR proteins in response to DNA strand breaks in yeast. Cell 97: 621-633.

McBride, A.E. and Silver, P.A. 2001. State of the arg: Protein methylation at arginine comes of age. Cell 106: 5-8.

McBride, A.E., Weiss, V.H., Kim, H.K., Hogle, J.M., and Silver, P.A. 2000 Analysis of the yeast arginine methyltransferase Hmtlp/Rmtlp and its in vivo function. Cofactor binding and substrate interactions. J. Biol. Chem. 275: 3128-3136.

Mellor, J. 2006. It takes a PHD to read the histone code. Cell 126: 22-24.

Mills, K.D., Sinclair, D.A., and Guarente, L. 1999. MEC1-dependent redistribution of the Sir3 silencing protein from telomeres to DNA double-strand breaks. Cell 97: 609-620.

Morillon, A., O'Sullivan, J., Azad, A., Proudfoot, N., and Mellor, J. 2003. Regulation of elongating RNA polymerase II by forkhead transcription factors in yeast. Science 300: 492-495.

Mowen, K.A., Tang, J., Zhu, W., Schurter, B.T., Shuai, K., Herschman, H.R., and David, M. 2001. Arginine methylation of STAT1 modulates IFN $\alpha / \beta$-induced transcription. Cell 104: 731-741.

Rusche, L.N., Kirchmaier, A.L., and Rine, J. 2003. The establishment, inheritance, and function of silenced chromatin in Saccharomyces cerevisiae. Annu. Rev. Biochem. 72: 481-516.

Shen, E.C., Henry, M.F., Weiss, V.H., Valentini, S.R., Silver, P.A., and Lee, M.S. 1998. Arginine methylation facilitates the nuclear export of hnRNP proteins. Genes \& Dev. 12: 679-691.

Strahl, B.D., Briggs, S.D., Brame, C.J., Caldwell, J.A., Koh, S.S., Ma, H., Cook, R.G., Shabanowitz, J., Hunt, D.F., Stallcup, M.R., et al. 2001 Methylation of histone $\mathrm{H} 4$ at arginine 3 occurs in vivo and is mediated by the nuclear receptor coactivator PRMT1. Curr. Biol. 11: 9961000.

Tamburini, B.A. and Tyler, J.K. 2005. Localized histone acetylation and deacetylation triggered by the homologous recombination pathway of double-strand DNA repair. Mol. Cell. Biol. 25: 4903-4913.

Wang, Y., Wysocka, J., Sayegh, J., Lee, Y.H., Perlin, J.R., Leonelli, L., Sonbuchner, L.S., McDonald, C.H., Cook, R.G., Dou, Y., et al. 2004 Human PAD4 regulates histone arginine methylation levels via demethylimination. Science 306: 279-283.

Yu, M.C., Bachand, F., McBride, A.E., Komili, S., Casolari, J.M., and Silver, P.A. 2004. Arginine methyltransferase affects interactions and recruitment of mRNA processing and export factors. Genes \& DeV. 18: 2024-2035. 


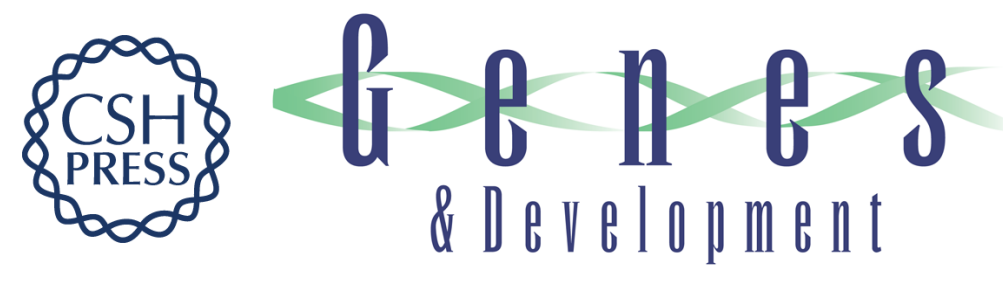

\section{The role of protein arginine methylation in the formation of silent chromatin}

Michael C. Yu, Dudley W. Lamming, Julian A. Eskin, et al.

Genes Dev. 2006, 20:

Access the most recent version at doi:10.1101/gad.1495206

References This article cites 34 articles, 22 of which can be accessed free at: http://genesdev.cshlp.org/content/20/23/3249.full.html\#ref-list-1

License

Email Alerting

Receive free email alerts when new articles cite this article - sign up in the box at the top Service right corner of the article or click here.

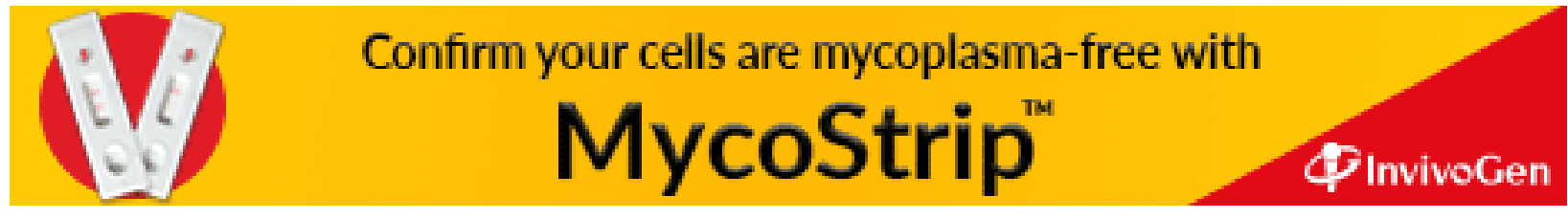

